\title{
Evaluación de la Arquitectura de Negocio a través del Análisis de Factores Críticos para el Desempeño de una Organización
}

\author{
Jorge E. Puerta-Ramírez, Jaime A. Giraldo-García y Mari L. Tabares-López \\ Universidad Nacional de Colombia, Facultad de Ingeniería y Arquitectura, Departamento de Ingeniería \\ Industrial, Campus La Nubia Bloque Q piso 2, Manizales - Colombia. \\ (e-mail: jepuertar@unal.edu.co, jaiagiraldog@unal.edu.co,mltabaresl@unal.edu.co)
}

Recibido Mar. 8, 2018; Aceptado May. 17, 2018; Versión final Jul. 27, 2018, Publicado Abr. 2019

\section{Resumen}

Se ha evaluado la arquitectura de negocio de la Contraloría General de la República de Colombia a través del análisis de factores que se consideran como críticos para su desempeño. Se identificaron factores críticos como el esquema organizacional y su medición en términos de adaptabilidad, efectividad y eficiencia. Para ello, se diseñó un instrumento y se aplicó el Análisis de Componentes Principales para reducir las dimensiones y poder obtener los factores que faciliten el análisis de la información acerca del estado actual de la Arquitectura de Negocio. Esto se hace de acuerdo a los lineamientos definidos en las fases del ciclo de la metodología ADM (Architecture Development Method). El estudio permite establecer un conjunto de condiciones para la representación del estado actual de acuerdo a los requerimientos de capacidad arquitectónica del ADM. Con base en los resultados se concluye que, a través del análisis de factores considerados como críticos para el desempeño de la organización, es posible evaluar el estado actual de la Arquitectura de Negocio.

Palabras clave: arquitectura de negocio; metodología ADM; análisis de componentes principales; desempeño organizacional

\section{Business Architecture Evaluation through Critical Factor Analysis for the Performance of an Organization}

\begin{abstract}
The Business Architecture of the General Comptroller of the Republic of Colombia has been evaluated through the analysis of the factors that are considered critical for its performance. The critical factors were identified as the organizational scheme and its measurement in terms of adaptability, effectiveness and efficiency. To do this, an instrument was designed and Principal Components Analysis was applied to reduce the dimensions and obtain the factors that facilitate the analysis of the information on the current state of the Business Architecture. This is done according to the lines defined in the phases of the ADM (Architecture Development Method) methodology cycle. The study allows establishing a set of conditions for the representation of the current state according to the architectural capacity requirements of the ADM. Based on the results, it is concluded that through the analysis of the factors considered critical for the performance of the organization, it is possible to evaluate the current state of the Business Architecture.
\end{abstract}

Keywords: business architecture; ADM methodology; principal component analysis; organizational performance 


\section{INTRODUCCIÓN}

Actualmente las organizaciones tienen la necesidad de evaluar su funcionamiento y comportamiento en términos de Arquitectura de Negocio, lo que se convierte en un insumo importante a la hora de tomar decisiones estratégicas y de realizar transformaciones a su estructura organizacional. Ante esto, autores como Geyer y Fourie (2015), argumentan que las organizaciones deben definir formalmente y documentar sus procesos de negocio con el fin de entenderlos y así permitir un mejoramiento continuo de su gestión. Situación que no debe ser ajena a la CGR (Contraloría General de la República), ya que la Constitución Política de Colombia de 1991 "la instituye como el máximo órgano de control fiscal en Colombia, para ejercer, en representación de la comunidad, la vigilancia de la gestión fiscal y de los particulares o entidades que manejan fondos o bienes de la Nación, procurando el buen uso de los recursos y bienes públicos y de contribuir a la modernización del estado, mediante acciones de mejoramiento continuo". (CGR, 2016). Por consiguiente la CGR tiene una serie de retos políticos, jurídicos y de gestión, siendo éste último un factor importante a la hora de demostrar su eficiencia y efectividad en sus funciones de control fiscal, que como lo afirma De Velasco (2010), lleva implícito los conceptos de objetivo y de mejora, los cuales se deben evaluar para que sean sostenibles en el tiempo.

Al respecto, Ordoñez y otros (2014) mencionan que la Arquitectura de Negocio en las organizaciones es normalmente modelada o creada por expertos, utilizando herramientas para el diseño de procesos de negocio en donde plasman las operaciones o tareas que se necesita ejecutar en la organización. Las organizaciones que pretenden diseñar, modelar o evaluar su Arquitectura de Negocio, tienen que empezar revisando grandes cantidades de información acerca de los procesos existentes. De la misma manera Acosta y Espino (2014) indican que el uso de técnicas para describir y analizar los procesos de negocios de una organización, permiten comprender el negocio antes de considerar alguna decisión y que dentro de las características que incluyen la mayoría de las técnicas de modelado de este tipo, se pueden mencionar los flujos de información y los diagramas de actividades. Señalan también éstos autores que aunque los modelos son fáciles de comprender y validar por las partes interesadas y proporcionan un buen punto de partida para soluciones tecnológicas, no describen el "por qué" de las situaciones problemáticas al interior de la organización.

Como consecuencia de lo anterior y teniendo en cuenta lo comentado por Crespo y Santos (2015), en cuanto a que la Arquitectura de Negocio, no solo describe como una organización opera funcionalmente, sino que también define y describe los procesos y objetivos de negocio necesarios para la implantación de la estrategia organizacional; y después de considerar las diferentes técnicas de Arquitectura de Negocio identificadas por Cuenca y otros (2008), Plattner (2014) y Bork (2014), tales como CIMOSA, GRAI-GIM, PERA, GERAM, ARIS, ZACHMAN y TOGAF, se seleccionó el marco de referencia TOGAF (The Open Group Architecture Framework), el cual incluye el Método de Desarrollo de la Arquitectura (ADM) que comprende: guías, técnicas y modelos de referencia, siendo éste el más completo y riguroso en la definición de los elementos de los procesos de negocio.

El ADM es un método flexible y como lo afirman Cabrera y otros (2015), permite su acoplamiento a las necesidades específicas de la organización, pero para ello debe realizarse un diagnóstico donde se identifique el estado actual desde varios aspectos o perspectivas, Cordero (2010) y Rivera (2016) las describen como la vista estratégica de la organización, la vista de procesos, la vista de información, la vista de aplicaciones y la vista tecnológica. Por el contrario, autores como Arango y otros (2010), se refieren a cuatro dominios (i) arquitectura de información; (ii) arquitectura de sistemas; (iii) arquitectura tecnológica; y (iv) arquitectura de negocio, siendo éste último el que se encarga de la descripción de la estructura organizacional, de los procesos de negocio, los sistemas de planeación y control, los mecanismos de gobierno y administración de políticas y procedimientos en el entorno de la organización.

Con el objetivo de realizar la evaluación de la Arquitectura de Negocio de la CGR, se diseñó y aplicó un instrumento que permitiera conocer el estado actual de los factores que se identificaron como críticos para el desempeño de la organización, tales como: el esquema organizacional, procesos y procedimientos, sistemas de información, adaptabilidad, efectividad y eficiencia, el cual fue validado por expertos y determinada su confiabilidad mediante el uso del coeficiente alfa de Cronbach. Para el análisis estadístico de los resultados se utilizó la técnica de Análisis de Componentes Principales, ya que como lo mencionan Navarro y Zamora (2012) y Manosalvas y otros (2015), es una herramienta idónea para éste estudio, la cual permite reducir las dimensiones y obtener los factores que faciliten el análisis de la información acerca del estado actual de la Arquitectura de Negocio de acuerdo a los lineamientos definidos en las fases del ciclo de la metodología ADM.

\section{METODOLOGÍA}

El desarrollo metodológico se presenta en dos etapas: en una se describe el marco de referencia de la arquitectura seleccionada para realizar la evaluación; y en otra etapa el diseño, validación, aplicación y 
Análisis de Componentes Principales del instrumento utilizado para la identificación de los factores críticos para el desempeño de la organización.

\section{Etapa 1. Marco de referencia de la arquitectura seleccionada}

Para la especificación de los dominios de arquitectura empresarial existen diferentes marcos de referencia, los cuales según Arango y otros (2010), determinan en qué términos se estructura dicha arquitectura. Así mismo Kosanke y otros (2015) argumentan que lo más importante de cualquier marco de referencia, es la capacidad para generar vistas del modelo de arquitectura y entre estos, Cuenca y otros (2008), Plattner (2014) y Bork (2014), mencionan los siguientes, según se muestra en la tabla 1.

Tabla 1: Marcos de referencia y sus vistas de arquitectura

\begin{tabular}{|l|l|}
\hline Marco de referencia & Vista de arquitectura \\
\hline CIMOSA & Vistas de Organización, Recursos, Información y Función. \\
\hline GRAI-GIM & Vistas del Sistema Físico, Decisional, de Información y Funcional. \\
\hline PERA & Arquitectura de Organización y RR.HH., del Sistema de Información y del Equipo de Producción. \\
\hline GERAM & Vistas de Organización, Recursos, Información y Función. \\
\hline ARIS & Vistas de Función, Datos, Organización y Control. \\
\hline Zachman & Datos, Función, Localización, Personas, Tiempo, Motivación. \\
\hline TOGAF & Visión Arquitectónica, Arquitectura de Negocio, del Sistema de Información y Tecnológica. \\
\hline
\end{tabular}

Para la investigación se seleccionó el marco de referencia TOGAF (The Open Group Architecture Framework), el cual incluye el Método de Desarrollo de la Arquitectura (ADM) que comprende: guías, técnicas y modelos de referencia, siendo éste el más completo y riguroso en la definición de los elementos de los procesos de negocio, además de cumplir con las características necesarias para el desarrollo metodológico, como las que menciona Santiago (2014), tabla 2.

Tabla 2: Características del marco de referencia TOGAF

\begin{tabular}{|l|l|}
\hline \multicolumn{1}{|c|}{ Característica } & \multicolumn{1}{c|}{ Descripción } \\
\hline Framework libre & $\begin{array}{l}\text { El framework es de uso gratuito para instituciones sin ánimo de lucro lo cual facilita el acceso al } \\
\text { mismo y su uso en el proyecto. }\end{array}$ \\
\hline $\begin{array}{l}\text { Documentación y } \\
\text { guías de apoyo }\end{array}$ & $\begin{array}{l}\text { Además de la especificación del framework para la construcción de la AE, cuenta con } \\
\text { documentación, artefactos y guías adicionales que facilitan la construcción de los productos de cada } \\
\text { fase definida en la metodología ADM. }\end{array}$ \\
\hline $\begin{array}{l}\text { Reconocimiento } \\
\text { internacional }\end{array}$ & $\begin{array}{l}\text { TOGAF es uno de los frameworks de mayor reconocimiento mundial como apoyo al desarrollo de la } \\
\text { arquitectura empresarial de una organización. }\end{array}$ \\
\hline $\begin{array}{l}\text { Framework } \\
\text { neutro e } \\
\text { integrable }\end{array}$ & $\begin{array}{l}\text { TOGAF ha sido desarrollado por el Open Group, por lo cual lo hace un framework neutro respecto a } \\
\text { herramientas y tecnologías a utilizar y está diseñado de tal manera que permite una fácil integración } \\
\text { con otros framework de AE y otras metodologías. }\end{array}$ \\
\hline
\end{tabular}

Tomando como base el marco de referencia de arquitectura empresarial TOGAF Versión 9.1 (2011), las especificaciones de la metodología ADM y lo descrito por Cerinza (2015), se identificaron las siguientes dimensiones para definir y limitar el alcance de la actividad de arquitectura para monitorear el desempeño organizacional, como se muestra en la tabla 3.

\section{Amplitud}

En cuanto a la extensión de la CGR para la actividad de arquitectura y debido a su tamaño, fig. 1; solo se tuvo en cuenta a los funcionarios pertenecientes a los grupos (tabla 4) de la Gerencia Colegiada del Departamento de Risaralda, el cual se encuentra ubicado en el centro occidente de la región andina de Colombia y es conocido por ser uno de los departamentos cafeteros del país, ya que gran parte de su economía se basa en la producción de dicho fruto.

\section{Profundidad}

El nivel de detalle del esfuerzo de arquitectura depende del proceso secuencial de identificación de los factores que se consideran como críticos para el desempeño de la organización y el Análisis de Componentes Principales para evaluar el estado actual de la Arquitectura de Negocio, fig. 2. 
Tabla 3: Dimensiones para limitar el alcance

\begin{tabular}{|l|l|}
\hline \multicolumn{1}{|c|}{ Dimensión } & \multicolumn{1}{c|}{ Consideraciones } \\
\hline Amplitud & $\begin{array}{l}\text { ¿Cuál es la extensión total de la empresa, y con qué parte de esa extensión debería tratar } \\
\text { el esfuerzo de arquitectura? }\end{array}$ \\
\hline Profundidad & ¿Qué nivel de detalle debería alcanzar el esfuerzo de arquitectura? \\
\hline Dominios de arquitectura & ¿Cuál o cuáles dominios de arquitectura se van a especificar? \\
\hline
\end{tabular}

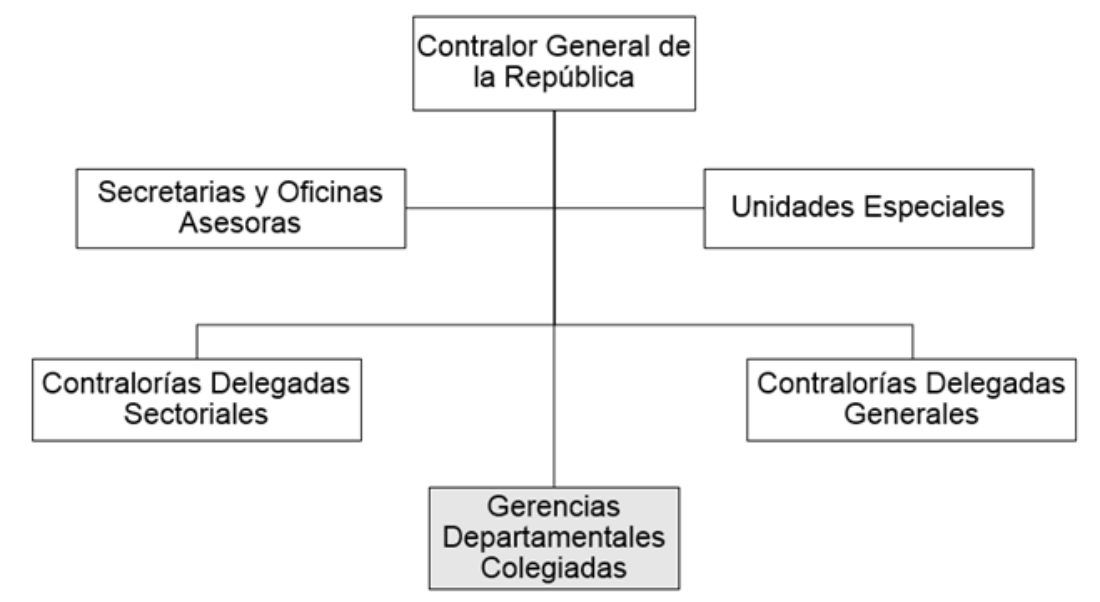

Fig. 1: Organigrama resumido de la Contraloría General de la República

Tabla 4: Distribución de los funcionarios por grupo (dependencia) en la Gerencia Departamental Colegiada de Risaralda de la CGR

\begin{tabular}{|l|c|c|}
\hline Grupo (dependencia) & No. Funcionarios por grupo & Porcentaje \\
\hline Vigilancia Fiscal & 45 & $58 \%$ \\
\hline Responsabilidad Fiscal & 11 & $14 \%$ \\
\hline Participación Ciudadana & 5 & $7 \%$ \\
\hline Apoyo a la Gerencia & 12 & $16 \%$ \\
\hline Colegiatura & 4 & $5 \%$ \\
\hline Total funcionarios & 77 & $100 \%$ \\
\hline
\end{tabular}

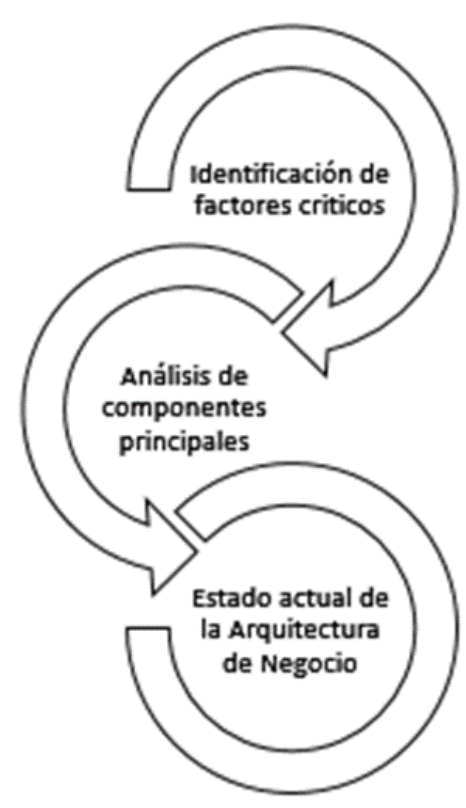

Fig. 2: Secuencia evaluación de la Arquitectura de Negocio 


\section{Dominios de arquitectura}

Teniendo en cuenta que el método de desarrollo de la arquitectura ADM es modular, lo que admite particionar las arquitecturas dentro de una misma empresa o áreas de ésta por dominios, fue elegido el dominio de Arquitectura de Negocio, para lo cual se debe seleccionar un instrumento que permita la identificación de los factores críticos para el desempeño de la organización.

De tal modo, la evaluación a través del análisis de factores críticos para el desempeño de la CGR se inició haciendo uso del dominio de Arquitectura de Negocio a nivel de diagnóstico, el cual implica realizar un análisis de la situación actual y un inventario de procesos de negocio. Adicional a lo anterior, en la publicación $\mathrm{CIO}$ del Ministerio de TI de Colombia (2013), se menciona que este diagnóstico debe incluir un análisis de integración e interoperabilidad entre procesos y subprocesos, fig. 3.

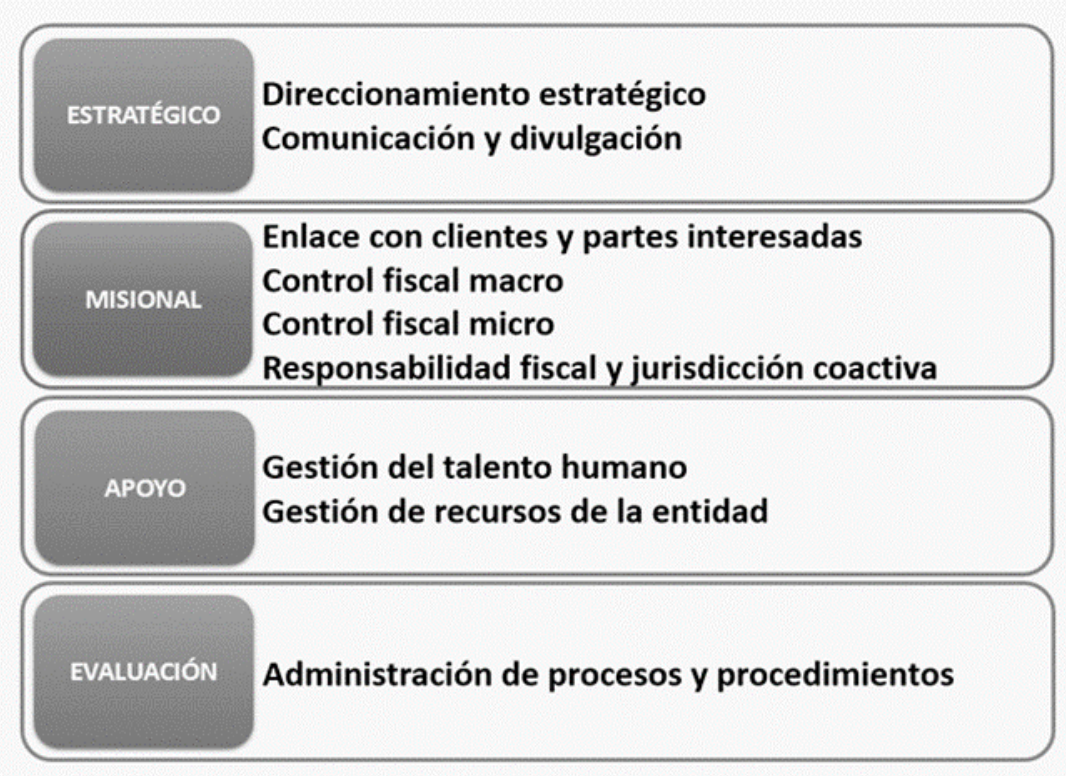

Fig. 3: Representación esquemática de la interrelación de los macroprocesos

\section{Etapa 2. Componentes Principales del instrumento}

Con base en investigaciones previas y los modelos diseñados y validados por instituciones de reconocido prestigio como el Departamento Administrativo de la Función Pública de Colombia (DAFP, 2015), Banco Interamericano de Desarrollo (BID, 2012) y Consorcio Internacional que desarrolla el Manual de Escala de Eficacia y Eficiencia Organizacional (OEES, 2015) y con el objetivo de evaluar la Arquitectura de Negocio de la CGR, se realizó la identificación de seis factores críticos para el desempeño de la organización, tabla 5; a partir de los cuales se diseñó un instrumento que consta de 63 preguntas, el cual fue aplicado a los funcionarios de la Gerencia Departamental Colegiada de Risaralda de la CGR.

Después de diseñar el instrumento, el cual fue validado por expertos y determinada su confiabilidad mediante el uso del coeficiente alfa de Cronbach, que como lo mencionan Bonett y Wright (2015) y González y Pazmiño (2015), es una forma sencilla y confiable para la validación del constructo de una escala y como una medida que cuantifica la correlación existente entre los ítems que componen ésta. Como se puede observar en la tabla 6, todos los valores para el coeficiente del alfa de Cronbach, son superiores a 0.7, resultado que como lo indica Bojórquez (2013) muestra una fuerte correlación entre cada uno de los factores que componen el instrumento y evidencia la validez del mismo.

Posteriormente, los datos fueron examinados con el software de análisis estadístico R, el cual aparte de ser de libre uso, ofrece una vasta variedad de métodos estadísticos, entre los que se incluyen según Navarro y Zamora (2012) y Manosalvas y otros (2015), las fases del Análisis de Componentes Principales, tabla 7; que es una técnica estadística de síntesis de la información, o reducción de la dimensión (número de variables). Es decir, ante un banco de datos con muchas variables, el objetivo será reducirlas a un menor número perdiendo la menor cantidad de información posible. Baró y Alemany (2000). Por lo antes expuesto, dicha técnica es ideal para reducir las dimensiones y poder obtener un acercamiento al estado actual de la arquitectura. 
Tabla 5: Factores críticos para el desempeño de la CGR

\begin{tabular}{|c|c|}
\hline Factor crítico & Descripción \\
\hline $\begin{array}{l}\text { Esquema } \\
\text { organizacional }\end{array}$ & $\begin{array}{l}\text { El esquema organizacional es la forma en que se determinan las relaciones entre las áreas } \\
\text { misionales y áreas de apoyo, y se definen las líneas de autoridad y responsabilidad, competencias } \\
\text { y funciones. }\end{array}$ \\
\hline $\begin{array}{l}\text { Procesos y } \\
\text { procedimientos }\end{array}$ & $\begin{array}{l}\text { Son entendidos como el quehacer en la organización y guardan relación directa con el desarrollo } \\
\text { de los planes, metas y objetivos y con la estructura orgánica de la entidad. No puede darse un } \\
\text { esquema de organización y el establecimiento de los planes sin que contemple el cómo } \\
\text { materializarlas. }\end{array}$ \\
\hline $\begin{array}{l}\text { Sistemas de } \\
\text { información }\end{array}$ & $\begin{array}{l}\text { El sistema de información se convierte en el instrumento para el control gerencial y la } \\
\text { retroalimentación a nivel interinstitucional e institucional a través de cada una de las dependencias } \\
\text { que integran la organización. Atender los principios de confiabilidad, oportunidad, integridad, } \\
\text { precisión y racionalidad, será la premisa de todo sistema de información en la medida en que esté } \\
\text { basado en objetivos institucionales. Los flujos de información se caracterizan por la recolección de } \\
\text { datos en forma racional permitiendo generar informes de gestión que permitan a sus ejecutivos } \\
\text { una eficiente toma de decisiones. }\end{array}$ \\
\hline Adaptabilidad & $\begin{array}{l}\text { Este factor considera las entidades que tienen la facilidad de adaptarse al cambio como respuesta } \\
\text { a las necesidades del entorno y de sus usuarios. Este tipo de factor permite dinamizar los procesos } \\
\text { y por ende los productos o servicios de la entidad, mediante estructuras planas y simples basadas } \\
\text { en el señalamiento de sus dependencias principales con niveles jerárquicos mínimos, dando } \\
\text { énfasis a las relaciones de colaboración, al trabajo en equipo, promoviendo el desarrollo del talento } \\
\text { humano, el autocontrol y la creatividad. }\end{array}$ \\
\hline Efectividad & Se define la efectividad como el grado en el que la organización es capaz de lograr sus metas. \\
\hline Eficiencia & $\begin{array}{l}\text { Se define la eficiencia como la relación entre los resultados logrados y los costos sufragados por } \\
\text { la organización para el cumplimiento de las metas. }\end{array}$ \\
\hline
\end{tabular}

Tabla 6: Valores obtenidos para el alfa de Cronbach

\begin{tabular}{|l|c|}
\hline \multicolumn{1}{|c|}{ Factor crítico } & Valor Alfa de Cronbach \\
\hline Esquema organizacional & 0.7906 \\
\hline Procesos y procedimientos & 0.9129 \\
\hline Sistemas de información & 0.8653 \\
\hline Adaptabilidad & 0.7657 \\
\hline Efectividad & 0.9333 \\
\hline Eficiencia & 0.8943 \\
\hline
\end{tabular}

Tabla 7: Fases Análisis de Componentes Principales. Basado en Navarro y Zamora (2012) y Manosalvas y otros (2015)

\begin{tabular}{|l|l|}
\hline \multicolumn{1}{|c|}{ Fase } & \multicolumn{1}{c|}{ Descripción } \\
\hline $\begin{array}{l}\text { Análisis de la } \\
\text { matriz de } \\
\text { correlaciones }\end{array}$ & $\begin{array}{l}\text { Un análisis de componentes principales tiene sentido si existen altas correlaciones entre las } \\
\text { variables, ya que esto es indicativo de que existe información redundante y, por tanto, pocos } \\
\text { factores explicarán gran parte de la variabilidad total. }\end{array}$ \\
\hline $\begin{array}{l}\text { Selección de los } \\
\text { factores }\end{array}$ & $\begin{array}{l}\text { La elección de los factores se realiza de tal forma que el primero recoja la mayor proporción posible } \\
\text { de la variabilidad original; el segundo factor debe recoger la máxima variabilidad posible no } \\
\text { recogida por el primero, y así sucesivamente. Del total de factores se elegirán aquéllos que recojan } \\
\text { el porcentaje de variabilidad que se considere suficiente. A éstos se les denominará componentes } \\
\text { principales. }\end{array}$ \\
\hline $\begin{array}{l}\text { Análisis de la } \\
\text { matriz factorial }\end{array}$ & $\begin{array}{l}\text { Una vez seleccionados los componentes principales, se representan en forma de matriz. Cada } \\
\text { elemento de ésta representa los coeficientes factoriales de las variables (las correlaciones entre } \\
\text { las variables y los componentes principales). La matriz tendrá tantas columnas como componentes } \\
\text { principales y tantas filas como variables. }\end{array}$ \\
\hline $\begin{array}{l}\text { Interpretación de } \\
\text { los factores }\end{array}$ & $\begin{array}{l}\text { Para que un factor sea fácilmente interpretable debe tener las siguientes características, que son } \\
\text { difíciles de conseguir: } \\
\text { - Los coeficientes factoriales deben ser próximos a 1. } \\
\text { - Una variable debe tener coeficientes elevados sólo con un factor. } \\
\text { - No deben existir factores con coeficientes similares. }\end{array}$ \\
\hline
\end{tabular}




\section{RESULTADOS Y DISCUSIÓN}

Al revisar los primeros resultados obtenidos con el software $\mathrm{R}$ a partir de los porcentajes de representatividad de las dimensiones de cada uno de los grupos de factores críticos analizados, tabla 8; se identifican los factores con menor representatividad a partir de la sumatoria entre las dimensiones 1 y 2 , determinando que dicho factor corresponde a la de adaptabilidad; por tanto, se prosigue a realizar una nueva sumatoria, pero en este caso entre las dimensiones 1 y 3 con el fin de determinar, igualmente, el factor menor representado, esto para explicar el factor adaptabilidad a través de este nuevo factor.

Tabla 8: Porcentajes de representatividad

\begin{tabular}{|l|c|c|c|c|c|c|c|}
\hline \multicolumn{1}{|c|}{ Factor crítico } & Dim.1 & Dim.2 & Dim.3 & Dim.4 & Dim.5 & Dim.1+Dim.2 & $\begin{array}{c}\text { Dim. } 1+ \\
\text { Dim.3 }\end{array}$ \\
\hline $\begin{array}{l}\text { Esquema } \\
\text { organizacional }\end{array}$ & 0.7285616 & 0.13573712 & 0.0106872 & 0.04385609 & 0.07404229 & 0,86429872 & 0,7392488 \\
\hline $\begin{array}{l}\text { Procesos y } \\
\text { procedimientos }\end{array}$ & 0.7329542 & 0.12412613 & 0.0854319 & 0.01226244 & 0.01251420 & 0,85708033 & 0,8183861 \\
\hline $\begin{array}{l}\text { Sistemas de } \\
\text { información }\end{array}$ & 0.6430500 & 0.21325770 & 0.0510371 & 0.05898309 & 0.03002852 & 0,8563077 & 0,6940871 \\
\hline Adaptabilidad & 0.7407957 & 0.08662109 & 0.0419917 & 0.09887955 & 0.03098271 & 0,82741679 & 0,7827874 \\
\hline Efectividad & 0.7236931 & 0.16600652 & 0.0054450 & 0.03098951 & 0.03213092 & 0,88969962 & 0,7291381 \\
\hline Eficiencia & 0.6549514 & 0.22807327 & 0.0738853 & 0.00270480 & 0.00001831 & 0,88302467 & 0,7288367 \\
\hline
\end{tabular}

Así pues, se puede decir, que para el caso de estudio el factor adaptabilidad, tendrá una tendencia de comportamiento similar al reflejado por el factor sistemas de información, esto debido a que una persona muestra mejor desempeño si conoce a plenitud los sistemas de información, además de saber cómo es el funcionamiento organizacional, jerárquico y procedimental de la organización, es decir, un funcionario es eficiente si está adaptado a su cargo y/o lugar de trabajo y tiene además la capacidad para asimilar nuevos conocimientos.

Del mismo modo se agrupan los factores con aquellos que tengan mayor asociación y por el grado de varianza que estén explicando, de tal manera que dichos factores se agrupan en 6 dimensiones y como se observa en la tabla 9, las dimensiones 1 y 2 representan el $86.297 \%$ de los datos, por lo tanto se trabajará con éstas dos.

Tabla 9: Porcentajes de representatividad

\begin{tabular}{|l|l|l|l|l|l|l|l|}
\hline Valores & Dim. 1 & Dim. 2 & Dim. 3 & Dim. 4 & Dim. 5 & Dim. 6 & Dim.1 + Dim. 2 \\
\hline Varianza & 4.224 & 0.954 & 0.268 & 0.248 & 0.180 & 0.126 & 5.178 \\
\hline \% de varianza & 70.400 & 15.897 & 4.475 & 4.128 & 2.995 & 2.105 & 86.297 \\
\hline \% de varianza acumulada & 70.400 & 86.297 & 90.772 & 94.900 & 97.895 & 100.000 & 86.297 \\
\hline
\end{tabular}

Así mismo el círculo de correlaciones, fig. 4; muestra que todos los factores presentan una correlación positiva, sin embargo, los factores sistemas de información, esquema organizacional y procesos y procedimientos, tienen una relación aún más estrecha, significándose esto que las personas que le dieron calificación de siempre o casi siempre a uno también lo hicieron con el otro, esto debido a que son dos aspectos que estructuralmente están muy ligados con el fin de promover el desempeño de la CGR; por otro lado los factores adaptabilidad, efectividad y eficiencia también muestran una buena correlación, esto debido a que la efectividad en la realización de las tareas depende en gran medida del conocimiento que tienen los funcionarios de sus funciones, actividades y desempeño organizacional, de la adaptación que tienen al cargo, a los puestos de trabajo, a los compañeros y a la organización como tal.

La correlación positiva más mínima que se da entre los factores sistemas de información y eficiencia, es decir, son dos aspectos que tienen características comunes pero no necesariamente el óptimo desempeño en uno conlleve a resultados positivos en el otro, esto debido a que a nivel de procesos no siempre es posible medir la eficiencia porque se pueden ver involucrados factores externos no controlables por la organización. En cuanto a los conglomerados, fig. 5; éstos presentan las agrupaciones que se formaron entre los funcionarios que ocupan diferentes cargos dentro de la CGR, pero que presentaron una tendencia de respuestas similar al momento de contestar el instrumento presentado, además la correlación que puede existir entre esta agrupación, y finalmente, la relación que tienen esos conglomerados con los factores evaluados. 


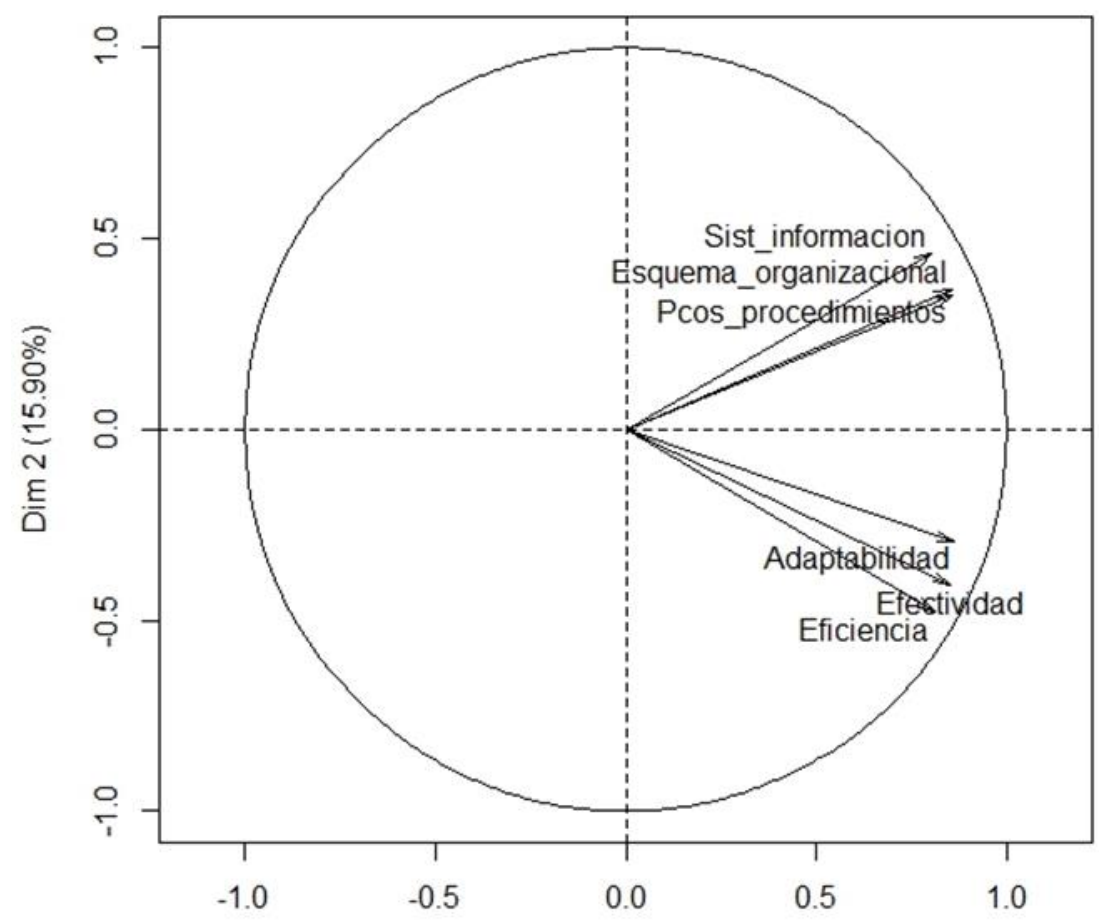

$\operatorname{Dim} 1(70.40 \%)$

Fig. 4: Círculo de correlaciones

En el clúster 1 se aglomeran los profesionales que calificaron como siempre y casi siempre las variables: procesos y procedimientos, estructura organizacional y sistemas de información, esto posiblemente porque son los cargos que se relacionan directamente con estos aspectos o que en el desarrollo de sus funciones le es indispensable el óptimo desempeño de éstos. Para el clúster 2 se agrupan funcionarios que ocupan los diferentes cargos dentro de la CGR (asistencial, directivo, ejecutivo, profesional y técnico) y representan las mejores calificaciones del cuestionario en cuanto a los factores: eficiencia, adaptabilidad y efectividad, los cuales constituyen características globales de la dirección estratégica de la CGR, por tanto, como equipo de trabajo al estar proyectados hacia el cumplimiento de un mismo objetivo estos factores significan herramientas clave para el desarrollo de las actividades propuestas.

\section{Factor map}

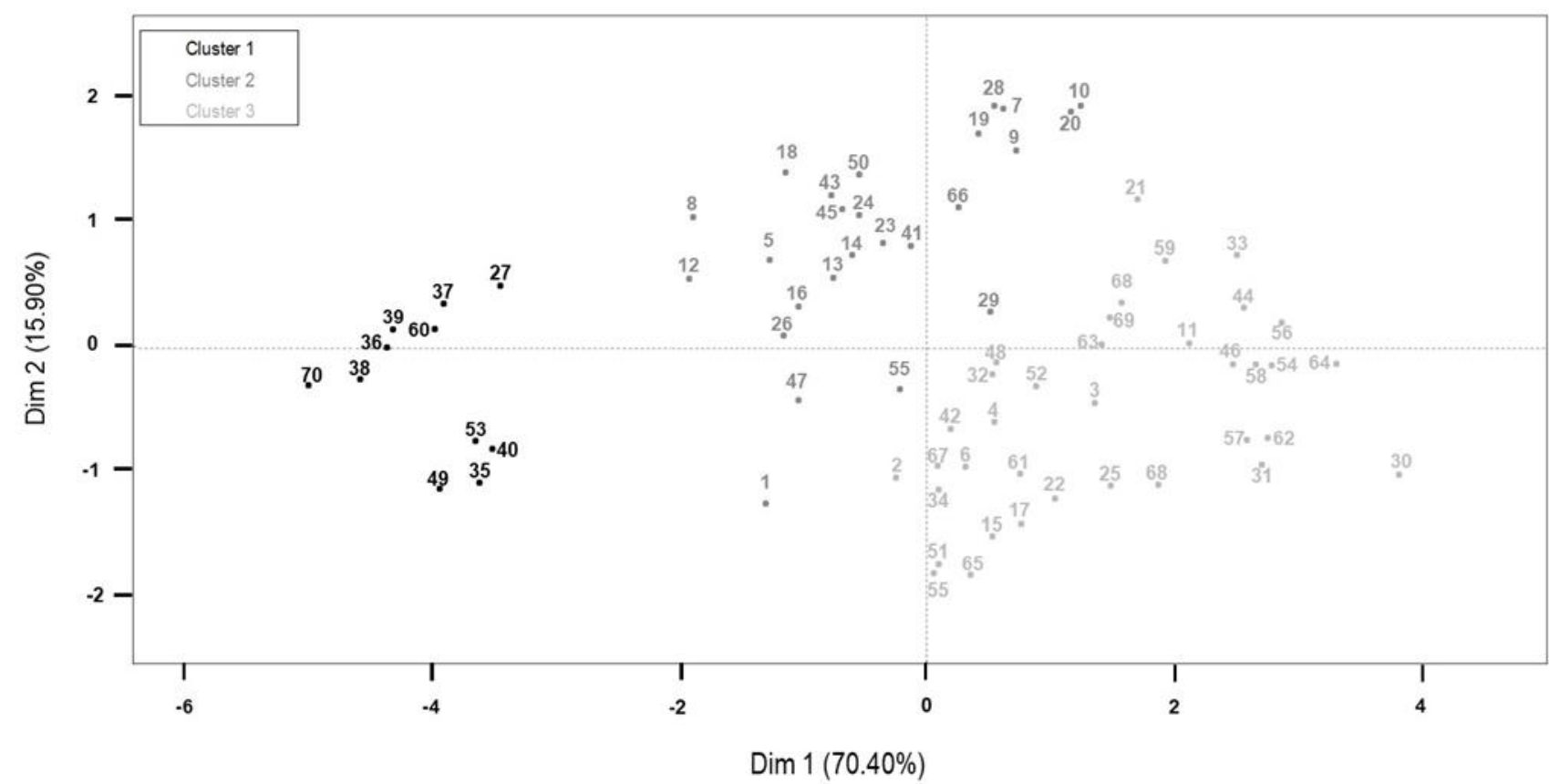

Fig. 5: Biplot de conglomerados 
En cuanto al clúster 3 se aglomeran los funcionarios de los cargos asistencial, profesional y técnico, y asistencial y profesional, respectivamente, quienes dieron calificaciones de nunca y casi nunca a las variables: para este mismo clúster, se aglomeran los profesionales que calificaron como siempre y casi siempre los factores: procesos y procedimientos, estructura organizacional y sistemas de información; mientras que para los factores: eficiencia, adaptabilidad y efectividad, la respuesta fue de a veces, mostrándose así una posición ambigua e indiferente frente a la evaluación del cuestionario, en este comportamiento pueden tener influencia situaciones como la antigüedad laboral o la situación de haber laborado antes en alguna otra dependencia u otro grupo de la CGR, pues normalmente, cuando no se tiene conocimiento de la estructura organizacional y de la manera en la que éstas operan, se tiende a mostrar un comportamiento impreciso e indeterminado.

Por lo anterior y partiendo de la idea que la Arquitectura de Negocio es un método para relacionar las metas y visiones de una organización con las operaciones que ésta realiza, IBM (2017) y que las organizaciones están estructuradas de acuerdo con sus flujos de trabajo y las interrelaciones entre los actores involucrados; al hacer un análisis de representatividad de las dimensiones de cada uno de los grupos de factores analizados en términos de adaptabilidad, efectividad y eficiencia, se puede identificar que la información acerca del estado actual de la Arquitectura de Negocio es fundamental, como soporte para el análisis de la CGR con miras al aumento del rendimiento de sus procesos como lo menciona Ortega y otros (2012). En conclusión al hacer un análisis en términos de ADM y recordando que el alcance del estudio solo abarca la evaluación del dominio de Arquitectura de Negocio a nivel de diagnóstico, los factores críticos se pueden agrupar en sistemas de información, esquema organizacional, procesos y procedimientos (Visión de Arquitectura), adaptabilidad, efectividad y eficiencia (Arquitectura de Negocio), fig. 6.

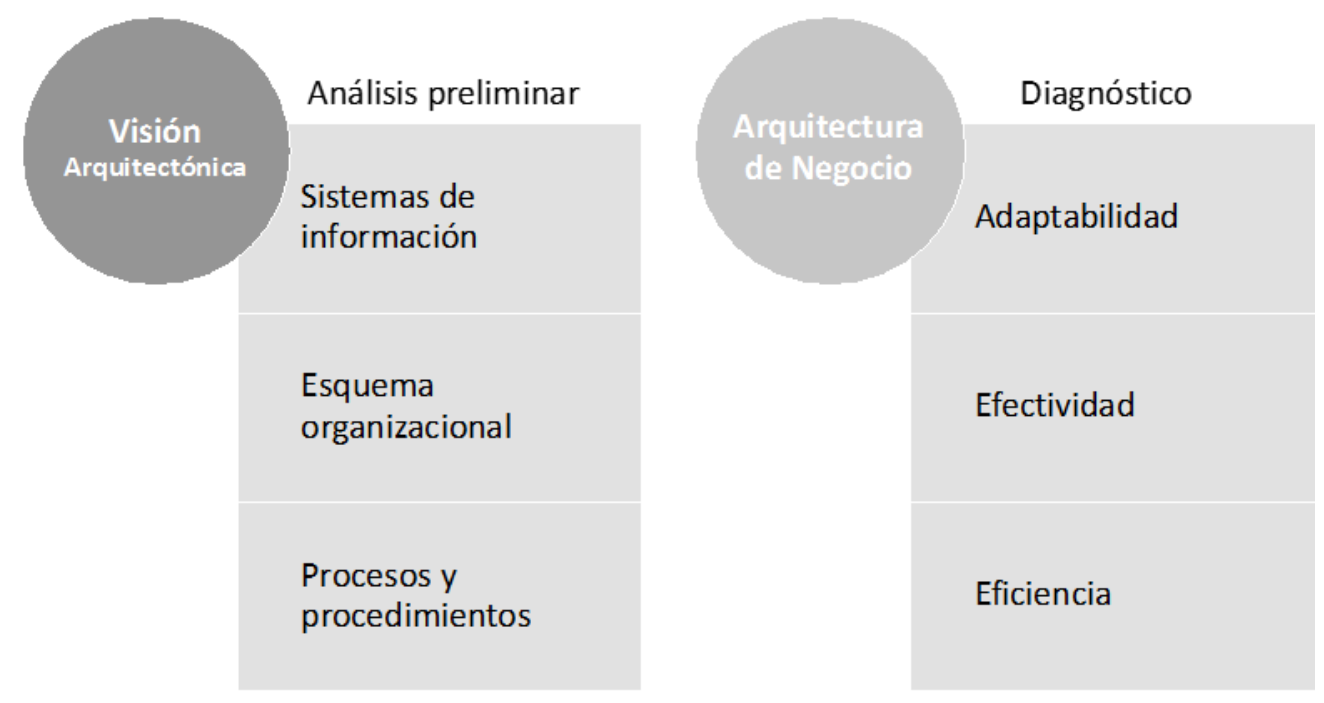

Fig. 6: Evaluación de Arquitectura de Negocio a partir de factores críticos

Finalmente al realizar una comparación entre los resultados obtenidos, con los de otros estudios ya publicados previamente en la literatura internacional y teniendo en cuenta que el tema central del trabajo es evaluar el estado actual de la Arquitectura de Negocio, se encuentra que AL-Ghamdi (2017) propone un modelo para medir el impacto de la Arquitectura de Negocio, pero este solo se concentra en evaluar los beneficios potenciales de su implementación sin haber hecho un diagnóstico de la situación actual de la organización. Por otro lado Cardwell (2010) en su estudio sobre la influencia de la Arquitectura Empresarial en los procesos de éxito, ya había evidenciado la necesidad de realizar una evaluación del estado actual antes de emprender cualquier esfuerzo de Arquitectura, pero solo lo hace por medio de diagramas de procesos y sin utilizar ninguna técnica estadística para validar lo plasmado en estos. Por último Nikpay y Ahmad (2017) presentan una metodología de implementación de Arquitectura Empresarial que busca identificar las prácticas y los factores efectivos a través de la aplicación de instrumentos a profesionales del área, no a una organización en particular como se hizo en el presente trabajo.

\section{CONCLUSIONES}

De los resultados expuestos en el punto anterior se pueden exponer las siguientes conclusiones:

(i) A partir del análisis estadístico de factores críticos, se evidenciaron las correlaciones positivas que existen entre estos, lo que permite establecer un conjunto de condiciones para la representación del estado actual de acuerdo a los requerimientos de capacidad arquitectónica de la metodología ADM. Con base en los resultados se concluye que a través del análisis de factores considerados como críticos para el desempeño de la 
organización, es posible evaluar el estado actual de la Arquitectura de Negocio; (ii) Si bien es cierto que las seis variables analizadas tienen un papel importante en el desempeño de la CGR, es clave la identificación de relaciones por factores que se realizó, pues esto permitió agruparlos por correlaciones y caracterizarlos según el impacto que tienen en la construcción de una Arquitectura de Negocio, específicamente los factores que aportan a la Visión de Arquitectónica y de Arquitectura de Negocio, tanto en su análisis preliminar como en su evaluación diagnóstica; (iii) Una contribución novedosa de la investigación, es la utilización de una metodología cuantitativa a través de la aplicación del Análisis de Componentes Principales mediante el tratamiento multidimensional, estableciendo coordenadas determinadas por la cercanía o lejanía de los factores considerados como críticos para el desempeño de la organización, dándole mayor validez a los resultados descriptivos que se utilizan normalmente en este tipo de evaluaciones de Arquitectura de Negocio; y (iv) Finalmente, se considera pertinente iniciar nuevas investigaciones donde no solamente se haga una evaluación del estado actual de la Arquitectura de Negocio, sino un análisis de brechas que permita proponer una nueva arquitectura para la CGR.

\section{AGRADECIMIENTOS}

Los autores agradecen a la Universidad Nacional de Colombia sede Manizales, por el apoyo institucional y financiero al desarrollo de esta investigación, la cual hace parte del proyecto "La Arquitectura Empresarial como Instrumento para Analizar la Incidencia de la Administración con Enfoque Burocrático en los Resultados del Control Fiscal Micro. Caso aplicado", código HERMES 35472.

\section{REFERENCIAS}

Acosta, A.P. y M.M. Espino, Modelos de requisitos basados en $\mathrm{i}^{\star}$ para detectar proactividad en dashboards, Rev. Lámpsakos, ISSN: 2145-4086, (12), 101-109 (2014)

AL-Ghamdi, A.S.A.M., A proposed model to measure the impact of business architecture, Cogent Business \& Management, 4(1), 1405493 (2017)

Arango, M.D., J. E. Londoño y J.A. Zapata, Arquitectura empresarial - una visión general, Rev. Ingenierías, ISSN: $1692-$ 3324, 9(16), 101-111 (2010)

Banco Interamericano de Desarrollo, Evaluación Organizacional, 2ª Ed., Centro Interamericano de Investigaciones, Ottawa, Canadá (2012)

Baró, J. y R. Alemany, Estadística II, Fundació per a la Universitat Oberta de Catalunya, Barcelona, España (2000)

Bonett, D.G. y T.A. Wright, Cronbach's alpha reliability: Interval estimation, hypothesis testing, and sample size planning, doi 10.1002/job.1960, Journal of Organizational Behavior, 36(1), 3-15 (2015)

Bojórquez, J.A., L. López, M. Hernández y E. Jiménez, Utilización del alfa de Cronbach para validar la confiabilidad de un instrumento de medición de satisfacción del estudiante en el uso del software Minitab. In $11^{\text {th }}$ Latin American and Caribbean Conference for Engineering and Technology, Cancún, México, 14-16 de Agosto (2013)

Bork, D. y H. G. Fill, Formal aspects of enterprise modeling methods: a comparison framework. In System Sciences (HICSS), 47 ${ }^{\text {th }}$ Hawaii International Conference on (pp. 3400-3409), IEEE, doi: 10.1109/HICSS.2014.422, Hawaii, USA, 69 de Enero (2014)

Cabrera, A., J. Carrillo y otros tres autores, Diseño y Validación de Arquitecturas de Aplicaciones Empresariales, RISTIRevista lbérica de Sistemas e Tecnologias de Informação, (SPE4), 79-91 (2015)

Cardwell, G., The influence of Enterprise Architecture and process hierarchies on company success, Total Quality Management, 19(1-2), 47-55 (2010)

Cerinza, D.R., Modelo para medir los beneficios de una arquitectura empresarial, Master dissertation, Tesis de Maestría, Escuela Colombiana de Ingeniería Julio Garavito (2015)

CGR - Contraloría General de La República, Control Fiscal Participativo, Economía Colombiana, Edición 315, enerofebrero (2016)

CIO, Ministerio de TI de Colombia, Arquitectura Empresarial, 2 (2013)

Cordero, R.N.L. e I.R. Salavert, Las vistas arquitectónicas de software y sus correspondencias mediante la gestión de modelos, Doctoral dissertation, Tesis Doctoral, Universidad Politécnica de Valencia (2010)

Crespo, P. y V. Santos, Construction of integrated business management systems for micro and small enterprises/Construcao de sistemas integrados de gestao para micro e pequenas empresas, RISTI (Revista Iberica de Sistemas e Tecnologias de Informacao), (15), 35-50 (2015)

Cuenca, L., A. Ortiz y A. Boza, Desarrollo de una Herramienta Software para la Vista de Información de la Arquitectura CIMOSA, Información Tecnológica, 19(3), 97-106 (2008)

Departamento Administrativo de la Función Pública - DAFP., Guía para la construcción y análisis de Indicadores de Gestión, Bogotá, Colombia (2015) 
Departamento Administrativo de la Función Pública - DAFP., Guía para la Administración del Riesgo, Bogotá, Colombia (2014) De Velasco, J.A., Gestión por procesos, 4ª Ed., 45-60, ESIC Editorial, Madrid, España (2010)

Geyer, R.W. y C.J. Fourie, Determining the suitability of a business process modelling technique for a particular application, ISSN: 1012-277X, Rev. South African Journal of Industrial Engineering, 26(1), 252-266 (2015)

González, A.J. y M Pazmiño, Cálculo e interpretación del Alfa de Cronbach para el caso de validación de la consistencia interna de un cuestionario, con dos posibles escalas tipo Likert, Revista Publicando, 2(1), $62-67$ (2015)

IBM, Visión general de IBM Rational System Architect, en línea, 13 de diciembre (2017)

Kosanke, K., F. Vernadat y M. Zelm, Means to enable enterprise interoperation: CIMOSA Object Capability Profiles and CIMOSA Collaboration View, Annual Reviews in Control, 39, 94-101 (2015)

Manosalvas, C.A., L.O. Manosalvas y J. Nieves, El clima organizacional y la satisfacción laboral: un análisis cuantitativo riguroso de su relación, AD-minister, (26), 5-15 (2015)

Navarro, J. C. L. y A. I. Zamora, La competitividad de la política comercial en la economía internacional a través del análisis de componentes principales, Investigación y Ciencia, 20(54) (2012)

Nikpay, F., R. B. Ahmad y otros tres autores, An effective Enterprise Architecture Implementation Methodology, Information Systems and e-Business Management, 15(4), 927-962 (2017)

OEES - Consorcio Internacional de Investigación sobre Prácticas Basadas en la Evidencia, Manual de la Escala de Eficacia y Eficiencia Organizacional, Salamanca, España (2015)

Ordoñez, H., J. C. Corrales y C. Cobos, MultiSearchBP: Entorno para búsqueda y agrupación de modelos de procesos de negocio, Polibits, (49), 29-38 (2014)

Ortega, D., E. Uzcategui y M. Guevara, EAIF: Un framework de arquitectura empresarial orientado a servicio en correspondencia con MDA, ISSN: 1316-4821, Rev. Universidad Ciencia y Tecnología, 16(62), 23-32 (2012)

Plattner, H., The impact of columnar in-memory databases on enterprise systems: implications of eliminating transactionmaintained aggregates, Proceedings of the VLDB Endowment, 7(13), 1722-1729 (2014)

Rivera, A.L., Importancia de la Documentación de la Arquitectura de Software para el Desarrollo de Sistemas: Caso Practico Sistema de Conciliación de Indeval, Master dissertation, Tesis de Maestría, Instituto Tecnológico y de Estudios Superiores de Monterrey, Campus Ciudad de México (2016)

Santiago, C. P., Propuesta de Arquitectura Empresarial para una universidad como un apoyo a su desarrollo frente a los retos del Siglo XXI, Twelfth LACCEI Latin American and Caribbean Conference for Engineering and Technology, 1-11, Guayaquil, Ecuador, 22 a 24 de julio (2014)

The Open Group, Togaf 9.1. - Gruía, Latin American Spanish, en línea (2011) 
